\title{
Electromagnetic and Neutrino Output from Magnetic Reconnection in Poynting Flux Dominated Jets
}

\author{
J. C. Rodríguez-Ramírez, ${ }^{a}{ }^{*}$ E. M. de Gouveia Dal Pino, ${ }^{a}$ R. Alves Batista ${ }^{b}$ and P. \\ Kushwaha $^{c}$ \\ ${ }^{a}$ São Paulo University, (IAG-USP), R. do Matão, 1226 - Cidade Universitaria, São Paulo-SP, Brazil. \\ ${ }^{b}$ Radboud University Nijmegen, Department of Astrophysics/IMAPP, The Netherlands. \\ ${ }^{c}$ Aryabhatta Research Institute of Observational Sciences (ARIES), Manora Peak, Nainital 263001, India \\ E-mail: juan.rodriguez@iag.usp.br
}

\begin{abstract}
Neutrino-emitting blazars may accelerate cosmic ray (CR) protons at the inner regions of the jet, where most of the magnetic energy is likely to be dissipated. In this picture, the spectrum of the neutrino output is shaped by the soft photon fields that the parent hadrons encounter before leaving the source. In this work, we build a lepto-hadronic emission model based on particle acceleration by magnetic reconnection along the jet propagation axis, in the transition from magnetically to kinetically dominated flow. We employ the reconnection striped jet model to obtain the jet properties at three characteristic emission regions and derive the associated electromagnetic and neutrino output. We also perform Monte Carlo simulations for the propagation of CR protons within the jet as an alternative method for calculating the neutrino flux. We apply this emission model to interpret the 2017 multi-messenger event from the blazar TXS 0506+056 and we also discuss applications of the model in the context of flat-spectrum radio quasars and BL Lac objects.
\end{abstract}

$37^{\text {th }}$ International Cosmic Ray Conference (ICRC 2021)

July 12 th - 23rd, 2021

Online - Berlin, Germany

\footnotetext{
${ }^{*}$ Presenter
} 


\section{Introduction}

Blazars are likely high energy (HE) neutrino factories [1]. However, so far the acceleration mechanism that energises the parent hadrons, as well as the location where the particle acceleration process takes place in the jet, are issues not yet established. Currently, magnetic reconnection [2,3] and diffusive shock acceleration (DSA) [4] are perhaps the most invoked mechanisms to energise the particles that produce the emission of blazars. In this contribution, we focus on the electromagnetic (EM) and neutrino output of blazar jets driven by magnetic reconnection. This study is particularly motivated by the recent test particle simulations of [5] in 3D relativistic magnetohydrodynamics (RMHD) jets which suggest that fast magnetic reconnection driven by kink instability turbulence in magnetically dominated jets [6] can accelerate cosmic-ray (CR) protons up to ultra-high-energies (UHEs).

The blazar emission is strongly influenced by the target radiation fields that surround the regions of particle acceleration. Usually, the radiation of the putative dusty torus (DT) and broad-line region (BLR) are considered as the relevant target radiation fields. In this work, we investigate the impact of these target fields on the neutrino and EM output from magnetically dominated blazar jets. To estimate the physical properties of the jet (such as the magnetic field, bulk Lorentz factor, and magnetic dissipation power) as a function of the distance from the central engine, we follow the reconnection striped jet model developed by [7, 8]. This model predicts the formation of magnetic field reversals along the inner, magnetically dominated region of the jet (see Figure 1), which allows, in principle, for fast magnetic reconnection development. Therefore, it may reproduce similar effects as those found in the 3D MHD simulations of relativistic jets with kink-driven turbulent fast reconnection [5, 6]. For this reason, we have adopted it to build the semi-analytical multi-messenger (MM) emission model described below.

The striped jet model predicts a jet bulk Lorentz factor $\Gamma_{\mathrm{j}}$ that always increases along the jet propagation axis to an asymptotic terminal value. On the other hand, the predicted magnetic dissipation power $P_{\text {diss }}$ peaks at a finite distance from the central engine (see Figure 1). For typical parameters of blazars the peak of the dissipation power predicted by this model is attained beyond the length scale of the DT. We then assume that the major contributions of the external radiation fields to the blazar emission take place in the jet at the BLR and DT length scales. Based on this assumption we build a lepto-hadronic emission model in which the blazar emission is a superposition of the MM output from three characteristic regions, namely the length scales of the BLR, the DT, and the location of the magnetic dissipation peak (see Figure 1). We employ the striped jet model to obtain the jet properties at these characteristic locations and derive the associated EM and neutrino output within a one-zone approximation in each region. Additionally, we also perform Monte Carlo simulations of the propagating CR protons as an alternative and more precise method to obtain the neutrino emission.

In the next section, we describe the general considerations for the target soft radiation fields and the jet properties based on the reconnection jet model. In Section 3 we apply our MM emission model to interpret the 2017 neutrino event from the blazar TXS 0506+056 and also to reproduce the blazar sequence and its associated neutrino output. 


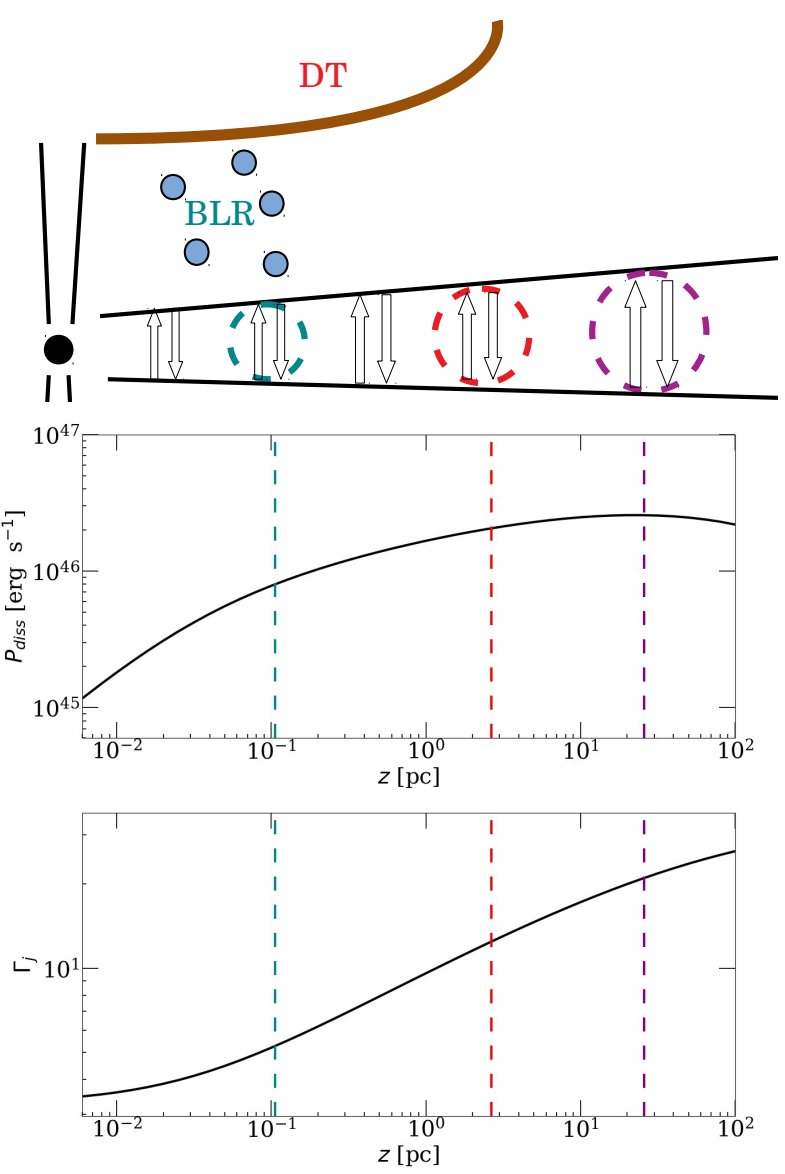

Figure 1: Top: Schematic representation of the three emission regions along the blazar jet defined by the length scale of the broad-line region (BLR), the dusty torus (DT), and the location of the magnetic dissipation peak (see the text.) The arrows represent the magnetic field reversals that allow for magnetic reconnection. Middle and Bottom: Magnetic reconnection power and jet bulk Lorentz factor as a function of the distance from the central engine as predicted by the striped jet model [7, 8]. The curves correspond to the model parameters of the SED of Figure 3.

\section{The properties of the blazar jet}

We consider an AGN accreting mass at a rate $\dot{M}_{\mathrm{a}}$ that launches two opposite directed jets with total mass loss rate $\dot{M}_{\mathrm{j}}$. We parameterise the total jet power $L_{\mathrm{j}}$ and the accretion disc luminosity $L_{\mathrm{AD}}$ in terms of the accretion rate in Eddington units $\dot{m}$, the terminal jet bulk Lorentz factor $\Gamma_{\infty}$, the ratio $\dot{\mu} \equiv \dot{M}_{\mathrm{j}} / \dot{M}_{\mathrm{a}}$, and the mass $M_{\bullet}$ of the central supermassive black hole (SMBH) as:

$$
L_{\mathrm{j}}=\Gamma_{\infty} \dot{\mu} \dot{m} \dot{M}_{\mathrm{E}}\left(M_{\bullet}\right) c^{2}, \quad L_{\mathrm{AD}}=\eta_{\mathrm{d}} \dot{m} \dot{M}_{\mathrm{E}}\left(M_{\bullet}\right) c^{2},
$$

where we fix the efficiency of the accretion disc luminosity as $\eta_{\mathrm{d}}=0.1$ and $M_{\mathrm{E}}=1.4 \times 10^{18}\left(M_{\bullet} / M_{\odot}\right)$ $\mathrm{g} \mathrm{s}^{-1}$ is the Eddington accretion rate onto the central engine. We estimate the size of the BLR and the DT with the scaling laws [9]:

$$
z_{\mathrm{BLR}}=10^{17} \mathrm{~cm}\left(\frac{L_{\mathrm{AD}}}{10^{45} \mathrm{erg} \mathrm{s}^{-1}}\right)^{1 / 2}, \quad z_{\mathrm{DT}}=2.5 \times 10^{18} \mathrm{~cm}\left(\frac{L_{\mathrm{AD}}}{10^{45} \mathrm{erg} \mathrm{s}^{-1}}\right)^{1 / 2} .
$$



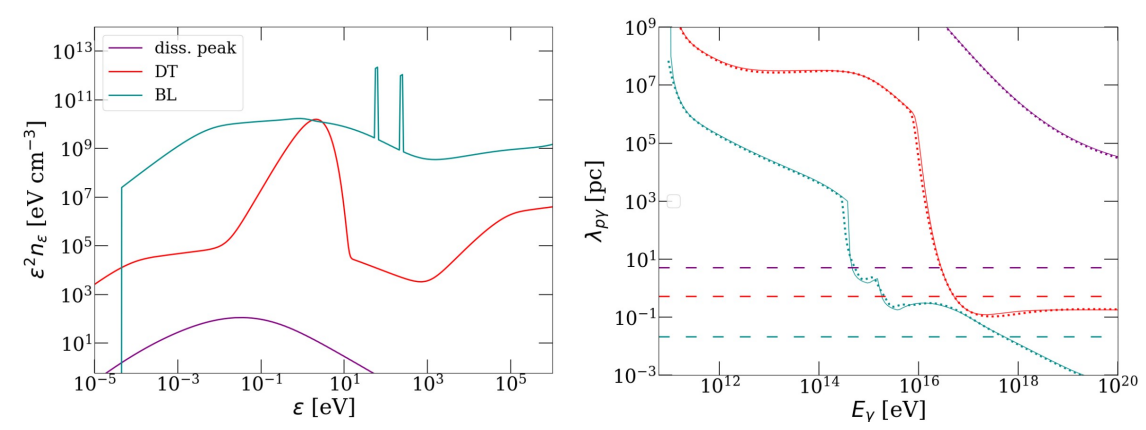

Figure 2: Left: Photon field densities in the jet co-moving frame at the three characteristic emission regions (see the text) and corresponding to the parameters of the SED model presented in Figure 3. Right: Mean free path curves of photo-hadronic interactions corresponding to the photon field densities displayed on the left. The continuous curves are obtained with the step-like approximation for the photo-meson cross-section described in [11], whereas the dotted curves are obtained with tabulated data for the photo-meson crosssection and using the CRPropa 3 tools ${ }^{1}$. The horizontal dashed lines indicate the size scale of each emission region.

For simplicity, we consider the BLR and DT radiation fields as isotropic and uniform within the radii given by equation 2 and non-effective as target fields outside these radii. In the rest frame of the SMBH, the DT photon field is assumed as given by a normalised black body with specific intensity

$$
I_{v, \mathrm{DT}}=\left(\frac{U_{\mathrm{DT}}}{U_{\mathrm{bb}}}\right) B_{\mathrm{bb}}\left(v, T_{\mathrm{DT}}\right) ; \quad U_{\mathrm{DT}}=\frac{L_{\mathrm{DT}}}{4 \pi c z_{\mathrm{DT}}^{2}},
$$

with a dust temperature of $T_{\mathrm{DT}}=500 \mathrm{~K}$. For the BLR photon field we consider constant specific intensities

$$
\Delta v I_{v, \mathrm{BLR}}=\frac{c}{4 \pi} U_{\mathrm{BLR}} ; \quad U_{\mathrm{BLR}}=\frac{L_{\mathrm{BLR}}}{4 \pi c z_{\mathrm{BLR}}^{2}},
$$

of energy bin widths $\Delta \log _{10}(E)=0.1$ centred at the H I and He II Ly $\alpha$ atomic lines energies 10.2 $\mathrm{eV}$ and $40.8 \mathrm{eV}$, respectively. Following [10], we assume the total DT luminosity of $L_{\mathrm{DT}}=0.5 L_{\mathrm{AD}}$ and the total BLR luminosity of $L_{\mathrm{BLR}}=0.1 L_{\mathrm{AD}}$ for the HI line and of $L_{\mathrm{BLR}}=0.05 L_{\mathrm{AD}}$ for the He II line.

In the left panel of Figure 2, we display an example of the target photon fields for photo-hadronic interactions as seen in the jet co-moving frame for each one of the aforementioned characteristic regions. Up to the BLR boundary, the target photon field is composed by photons from the BRL and the DT as well as by photons produced within the jet, namely, synchrotron and inverse Compton (IC) radiation, being the latter conformed by synchrotron self-Compton (SSC) plus external Compton (EC). At the location corresponding to DT size, we neglect BLR photons since they impinge this region from behind and thus we assume the target photons as conformed by the external DT photons and the internal jet radiation (synchrotron plus IC photons). With a similar argument, external photons are neglected at the location of the magnetic dissipation peak where we only consider internal jet radiation as the target field. This approximation is valid provided the peak of magnetic dissipation is well beyond the DT length scale, which is the case for all the models considered in this work.

\footnotetext{
1https://github.com/CRPropa/CRPropa3-data
} 
Following [7, 8], we estimate the magnetic field strength, the magnetic reconnection power, and the bulk Lorentz factor of the jet in each one of the characteristic emission regions through the following equations:

$$
\frac{\tilde{B}^{2}}{4 \pi}=\frac{4 \epsilon^{2}}{\Gamma_{\infty}^{6}\left(l_{\min } \theta_{\mathrm{j}} \zeta\right)^{2} \pi c} \frac{1-\chi(\zeta)}{\chi^{2}(\zeta)} L_{\mathrm{j}}, \quad P_{\mathrm{diss}}=\left[\frac{1-\chi(\zeta)}{\chi}\right]^{k} \zeta L_{\mathrm{j}}, \quad \frac{d \chi}{d \zeta}=\frac{(1-\chi(\zeta))^{k}}{\chi^{2}(\zeta)}
$$

where $\chi(\zeta) \equiv \Gamma_{\mathrm{j}} / \Gamma_{\infty}, \tilde{B}$ is the jet magnetic field in the jet co-moving frame, $\epsilon=0.1$ is the reconnection rate, $\zeta \equiv(2 \epsilon z) /\left(l_{\min } \Gamma_{\infty}^{2}\right), \theta_{\mathrm{j}}$ is the opening angle of the jet assumed as conical, and $l_{\min }=100 G M_{\bullet} / c^{2}$. We plot in Figure 1 the magnetic dissipation power and the jet bulk Lorentz factor as a function of the jet propagation axis given by the equations above and corresponding to a SMBH of mass $M_{\bullet}=3 \times 10^{9} \mathrm{M}_{\odot}$ and jet terminal bulk Lorentz factor of $\Gamma_{\infty}=32.8$.

\section{Electromagnetic and neutrino output}

Following [7], we assume that a small fraction of the magnetic dissipation power energises nonthermal particles accelerated by magnetic reconnection [5]. Under this assumption, we normalise the energy density $\tilde{U}_{\mathrm{p}, \mathrm{e}}$ of primary protons and electrons in the jet co-moving frame as

$$
\eta_{\mathrm{p}, \mathrm{e}} P_{\mathrm{diss}}=\left(\Gamma_{\mathrm{j}} \theta_{\mathrm{j}} z\right)^{2} \pi c \tilde{U}_{\mathrm{p}, \mathrm{e}}
$$

with $\eta_{\mathrm{p}, \mathrm{e}} \ll 1$, and $\tilde{U}_{\mathrm{p}, \mathrm{e}} \equiv \int \mathrm{d} E E \tilde{N}_{\mathrm{p}, \mathrm{e}}$. For CR electrons, the particle distribution $\tilde{N}_{\mathrm{e}}$ (particles per unit volume, per unit energy) is obtained by solving the stationary transport equation. For CR protons the particle distribution is obtained as $\tilde{N}_{\mathrm{p}}=\tilde{Q}_{\mathrm{p}} \Delta t$, where $\Delta t=\min \left\{t_{\mathrm{esc}}, t_{\text {cool }}(E)\right\}$, being $t_{\mathrm{esc}}=\theta_{\mathrm{j}} z / c$, and $t_{\text {cool }}^{-1}$ the total cooling rate due to proton synchrotron radiation, photopion production, and photo-pair production (Bethe-Heitler process). The injection function of CR protons $\tilde{Q}_{\mathrm{p}}$ (particles per unit energy, per unit time, per unit volume) is parameterised as a power-law (PL) with exponential cut-off, where the maximum energy is obtained by balancing the magnetic reconnection acceleration rate with the proton energy loss rates. A similar injection function is assumed for the primary relativistic electrons for which we additionally consider a smooth, broken PL.

We then build a lepto-hadronic emission model where primary electrons accounts for most of the EM blazar spectrum and CR protons, through internal cascades initiated by hadronic interactions, provide neutrino output and a subdominant contribution to the EM spectrum. To account for the EM radiation of hadronic origin we compute the emission within the jet from: (i) charged pion cascade, (ii) neutral pion cascade, (iii) Bethe-Heitler cascade, and (iv) proton synchrotron. To evaluate the internal gamma-ray absorption we consider that same target photon field that leads to photo-hadroic interactions. To evaluate the gamma-ray attenuation due to the extragalactic background light (EBL), we consider the Domínguez et al. [12] EBL model. Note, however, that other propagation effects such as those of intergalactic magnetic fields [13], not considered here, can significantly modify our SED models at the gamma-ray energies.

We display in Figure 3 an example of the MM blazar SED model described in this work. The dashed curves in different colours represent the total EM flux (including leptonic and hadronic components) produced in a given emission region, and the dotted curves are the respective neutrino 


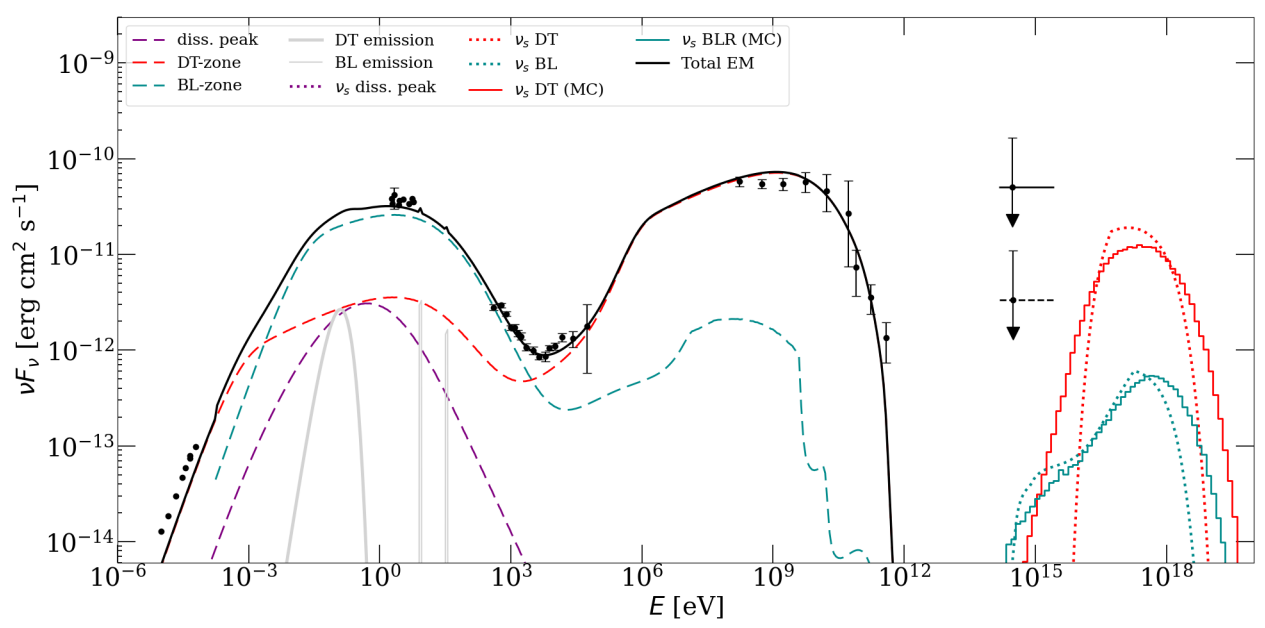

Figure 3: Blazar lepto-hadronic SED emission model based on the magnetic reconnection striped jet model (see the text). Curves above $\sim \mathrm{PeV}$ energies are neutrino fluxes. The dotted curves are obtained with analytic homogeneous approximation and the step-like curves are obtained with Monte Carlo emission calculation during 3D propagation of CR protons employing the CRPropa3 code (see the text). The overplotted data corresponds to the simultaneous electromagnetic and neutrino measure of the TXS $0506+056$ blazar reported in [1].

fluxes. This SED model is obtained assuming a SMBH of mass $M_{\bullet}=3 \times 10^{9} \mathrm{M}_{\odot}$, accreting at a rate of $\dot{m}=0.028$ with a jet producing CR protons and electrons that represent the fractions $\eta_{\mathrm{p}}=0.1$ and $\eta_{\mathrm{e}}=6 \times 10^{-4}$, respectively, of the magnetic reconnection power (see equation 6) in the characteristic emission regions.

We note that magnetic reconnection at the BLR length scale provides the main contribution to the blazar low energy bump whereas the emission produced at the DT length provides the main contribution to the blazar second bump. The emission produced at the magnetic dissipation peak is negligible in all EM and neutrino emission bands when compared to the contribution from the other regions. The radiation of the hadronic cascades contribute modestly to the high energy tail of the EM spectrum as well as to the X-ray emission bands. The overplotted data in Figure 3 is taken from [1] which corresponds to the 2017 simultaneous MM signal associated to the balzar TXS 0506+056.

In addition to the afore-described lepto-hadronic emission calculations, we performed Monte Carlo (MC) simulations employing the CPropa3 code [14] for the propagation and interaction of CR protons within the jet emission regions. To set the simulation environment, we use the jet physical properties, inferred from the semi-analytic SED emission model. We set a random magnetic field background with a turbulence spectrum of index -11/3 (generated with the CRPropa3 code) and an RMS strength equivalent to the magnetic field given by the semi-analytic model. This turbulent magnetic spectrum is compatible with the 3D RMHD jet simulations of [6] and [5] employed to calculate the acceleration of CRs by $3 \mathrm{D}$ magnetic reconnection. In these works, the jets undergo fast magnetic reconnection triggered by a turbulent magnetic field that approaches a Kolmogorov-like profile [6]. The mean free paths of photo-hadronic collisions employed in our MC simulation are calculated as described in Figure 2. The neutrino fluxes that result from this MC approach are 


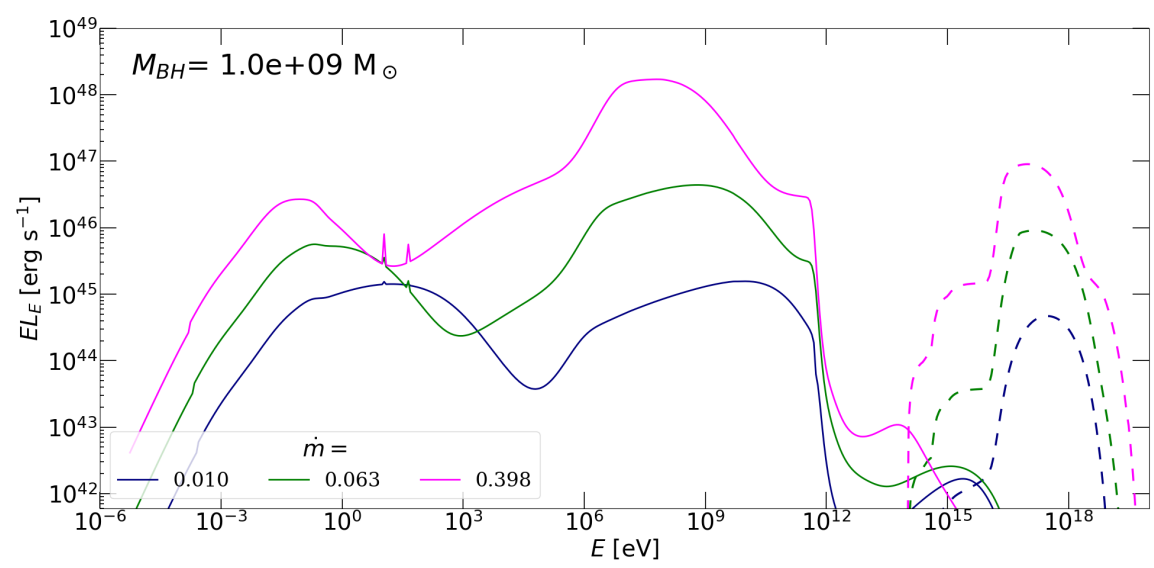

Figure 4: Blazar sequence trend derived from the magnetic reconnection, lepto-hadronic jet emission model described in the text. The different curves correspond to different accretion rates onto the central black hole in Eddington units as indicated in the legends. The blazar sequence from BL Lacs to FSRQs is reproduced as the peaks of the SED models with increasing accretion rate shift from right to left, respectively. The solid curves represent the total electromagnetic luminosity in the cosmic frame, whereas the dashed curves are the associated neutrino emission.

plotted with the step-like curves in Figure 3. In order to obtain a more realistic MC simulation of neutrino production, we aim to perform numerical radiative transfer to derive the target radiation fields directly from the RMHD simulated jet. This ongoing investigation will be the subject of a forthcoming publication.

Finally, we note that the lepto-hadronic jet emission model based on magnetic reconnection acceleration discussed in these proceedings can be applied to interpret the emission of BL Lacs and flat-spectrum radio quasars. In Figure 4, we reproduce the so-called blazar sequence trend as a function of the dimensionless accretion rate $\dot{m}$ onto the central engine. To obtain the blazar trend of Figure 4, we impose a dependence of the model parameters on $\dot{m}$ as $\dot{\mu} \equiv \dot{M}_{j} / \dot{M}_{a} \propto \dot{m}^{-1 / 2}$, $E_{b} \propto \dot{m}^{-1}$ (the electron energy break), and $\Gamma_{\infty} \propto \dot{m}^{1 / 4}$ (the terminal jet Lorentz factor).

\section{Acknowledgments}

We thank Konstancja Satalecka for useful comments. We acknowledge support from the Brazilian Funding Agency FAPESP (JCRR's grant: 2017/12188-5, EMGP's grant: 2013/105595) and PK's ARIES Aryabhatta Fellowship (AO/A-PDF/770). RAB acknowledges the financial support from the Radboud Excellence Initiative.

\section{References}

[1] IceCube Collaboration, M.G. Aartsen, M. Ackermann, J. Adams, J.A. Aguilar, M. Ahlers et al., Multimessenger observations of a flaring blazar coincident with high-energy neutrino IceCube-170922A, Science 361 (2018) eaat1378 [1807.08816]. 
[2] E.M. de Gouveia dal Pino and A. Lazarian, Production of the large scale superluminal ejections of the microquasar GRS 1915+105 by violent magnetic reconnection, A\&A 441 (2005) 845.

[3] D. Giannios, UHECRs from magnetic reconnection in relativistic jets, MNRAS 408 (2010) L46 [1007. 1522].

[4] J.G. Kirk, F.M. Rieger and A. Mastichiadis, Particle acceleration and synchrotron emission in blazar jets, A\&A 333 (1998) 452 [astro-ph/9801265].

[5] T.E. Medina-Torrejón, E.M. de Gouveia Dal Pino, L.H.S. Kadowaki, G. Kowal, C.B. Singh and Y. Mizuno, Particle Acceleration by Relativistic Magnetic Reconnection Driven by Kink Instability Turbulence in Poynting Flux-Dominated Jets, ApJ 908 (2021) 193 [2009. 08516].

[6] L.H.S. Kadowaki, E.M. de Gouveia Dal Pino, T.E. Medina-Torrejón, Y. Mizuno and P. Kushwaha, Fast Magnetic Reconnection Structures in Poynting Flux-dominated Jets, ApJ 912 (2021) 109 [2011.03634].

[7] H. Zhang and D. Giannios, Radiation signatures from striped blazar jet, MNRAS 502 (2021) 1145 [2012. 15189].

[8] D. Giannios and D.A. Uzdensky, GRB and blazar jets shining through their stripes, MNRAS 484 (2019) 1378 [1805.09343].

[9] G. Ghisellini and F. Tavecchio, The blazar sequence: a new perspective, MNRAS 387 (2008) 1669 [0802.1918].

[10] K. Murase, Y. Inoue and C.D. Dermer, Diffuse neutrino intensity from the inner jets of active galactic nuclei: Impacts of external photon fields and the blazar sequence, PRD 90 (2014) 023007 [1403.4089].

[11] A.M. Atoyan and C.D. Dermer, Neutral Beams from Blazar Jets, ApJ 586 (2003) 79 [astro-ph/0209231].

[12] A. Domínguez, J.R. Primack, D.J. Rosario, F. Prada, R.C. Gilmore, S.M. Faber et al., Extragalactic background light inferred from AEGIS galaxy-SED-type fractions, MNRAS 410 (2011) 2556 [1007 . 1459].

[13] A. Saveliev and R. Alves Batista, The intrinsic gamma-ray spectrum of TXS 0506+056: intergalactic propagation effects, MNRAS 500 (2021) 2188 [2009.09772].

[14] R. Alves Batista, A. Dundovic, M. Erdmann, K.-H. Kampert, D. Kuempel, G. Müller et al., CRPropa 3 a public astrophysical simulation framework for propagating extraterrestrial ultra-high energy particles, JCAP 5 (2016) 038 [1603.07142]. 